\title{
Study of Energetic Ion Generation from High- Intensity-Laser Dense-Plasma Interactions
}

\author{
K. Flippo ${ }^{\mathrm{a}}$, A. Maksimchuk ${ }^{\mathrm{a}}$, S. Banerjee ${ }^{\mathrm{a}}$, K. Nash ${ }^{\mathrm{a}}$, V. Wong ${ }^{\mathrm{a}}$, T. Lin ${ }^{\mathrm{a}}$, \\ K. Nemoto ${ }^{b}$, V. Yu. Bychenkov ${ }^{c}$, Y. Sentoku ${ }^{d}$, G. Mourou ${ }^{a}$, \\ D. Umstadter ${ }^{\mathrm{a}}$ \\ ${ }^{a}$ FOCUS Center, University of Michigan, Ann Arbor, MI 48109-2099, USA \\ ${ }^{b}$ CRIEPI, 2-11-1, Iwado-kita, Komae-shi, Tokyo, 201-8511 Japan \\ ${ }^{c}$ P. N. Lebedev Physics Institute, Russian Academy of Science, 117924 Moscow, Russia \\ ${ }^{d}$ Osaka University, Osaka Japan (Currently with GA, San Diego)
}

\begin{abstract}
We report on the characteristics of an ultrafast-laser driven proton beam from thinfilm targets. The difference in proton beam profiles, beam energies, and laser induced back ablation plumes between a dielectric (Mylar) and a conductor (aluminum) are discussed. Evidence for front-side acceleration and a method for beam manipulation are also presented.
\end{abstract}

\section{IONS FROM HIGH-INTENSITY-LASERS}

Since the discovery of forward accelerated protons and ions from high-intensitylaser thin-film interactions ${ }^{1,2,3}$, many groups have become interested in using these protons for a wide variety of applications, including: plasma diagnostics ${ }^{4}$, radiography ${ }^{5}$, radiation therapy ${ }^{6}$, radioisotope production $^{7,8}$, fast ignition fusion ${ }^{9}$, table-top nuclear reactions ${ }^{10}$ and nuclear physics ${ }^{11}$, and even space propulsion ${ }^{12}$. For these proton beams to become usefully wide-spread their origin must first be carefully understood and then their characteristics controlled.

In our previous experiments ${ }^{2}$ we used the second harmonic light from a $\mathrm{T}^{3}$ hybrid Ti:Sapph/Nd:Glass laser system producing 10TW. These initial experiments were limited to $2 \mathrm{~J}$ and $2 \times 10^{18} \mathrm{~W} / \mathrm{cm}^{2}$ due to non-linear effects in the doubling crystal. We observed that a collimated beam of protons, $40^{\circ}$ in divergence, and up to $1.5 \mathrm{MeV}$ could be accelerated forward through the target. The protons originate from a few tens to hundreds of Angstroms thick contamination layer on the surface of the target, consisting of mainly water vapor.

In a subsequent experiment ${ }^{8}$ we observed, using $6 \mathrm{~J}$ of the laser's fundamental frequency, at intensities up to $5 \times 10^{18} \mathrm{~W} / \mathrm{cm}^{2}$, that a beam of up to $10 \mathrm{MeV}$ was

CP647, Advanced Accelerator Concepts: Tenth Workshop, edited by C. E. Clayton and P. Muggli

(C) 2002 American Institute of Physics 0-7354-0102-0/02/\$19.00 


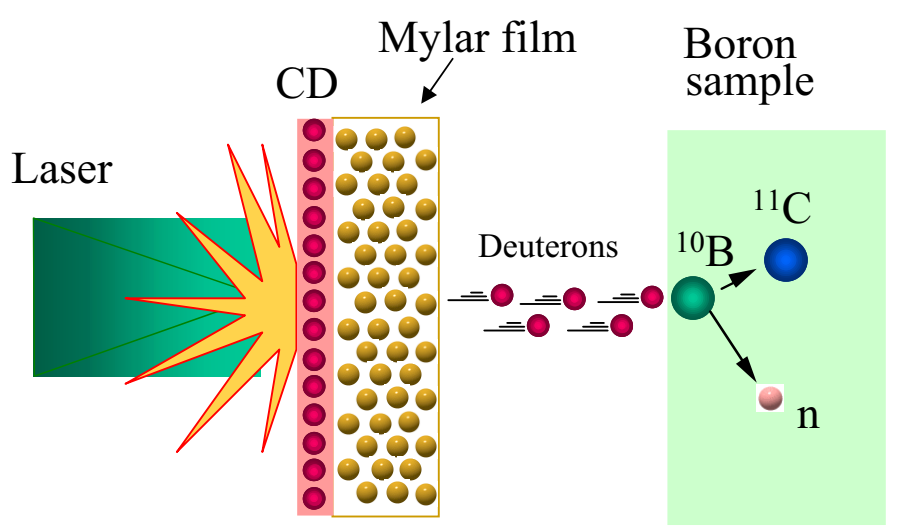

FIGURE 1. Setup for $10 \mathrm{~B}(\mathrm{~d}, \mathrm{n}) 11 \mathrm{C}$ nuclear activation experiments.

possible. We also showed that the majority of protons in our experiments originated from the front surface of the target through an experiment involving nuclear activation.

The experiment pictured in Fig. 1 consisted of laying down a very thin layer of deuterated plastic over a thin (6 micron) Mylar film. A sample of ${ }^{10} \mathrm{~B}$ was placed behind the target (in the forward direction), then the Mylar was irradiated by the laser either with the coated side or uncoated side facing the boron. Activation, via the reaction ${ }^{10} \mathrm{~B}(\mathrm{~d}, \mathrm{n}){ }^{11} \mathrm{C}$, was seen only when the deuterated surface was irradiated.

The front surface acceleration was explained by $\mathbf{v} \times \mathbf{B}^{13}$ or Brune $1^{14}$ heating of the electrons and acceleration into the solid. This produces a large electrostatic field which accelerates the protons into the target. Ponderomotive forces alone could not explain the needed electron temperatures for the observed proton energies assuming the electrostatic potential imparts energy to the ions as $\mathrm{E}_{\max }=C Z \sqrt{\lambda I}$, where $\mathrm{Z}$ is the atomic number, $\lambda I$ is the product of the laser wavelength and the intensity, and $C$ is the proportionality constant.

This explanation is only valid for a laser-solid interaction in which there is a high laser contrast between the foot of the pulse (pre-pulse) and the main pulse. In our experiment using the second harmonic the laser contrast was approximately $10^{7}: 1$, which did not produce a significant pre-plasma (1 micron or less), with which the highest intensity portion of the pulse could interact.

However, experiments at the fundamental frequency had a contrast of only $10^{5}: 1$, and a pre-plasma from several to ten microns could form. The formation of the preplasma is critical to acceleration of ions, because of the increase in absorbed laser radiation.

Hatchet $e t$. al. and Snavely et. al. ${ }^{2}$ have done experiments which they interpret as having protons which originated on the back surface, and in fact have shown via PIC simulation ${ }^{15}$ that both surfaces produce protons. Controlling which surface produces the beam of protons may lead to applications for each method. 


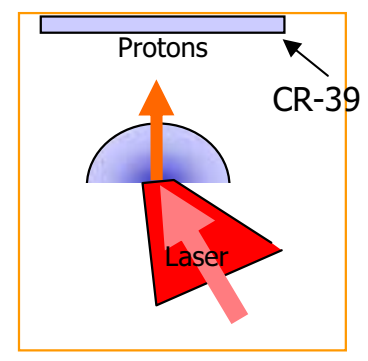

(a) Flat

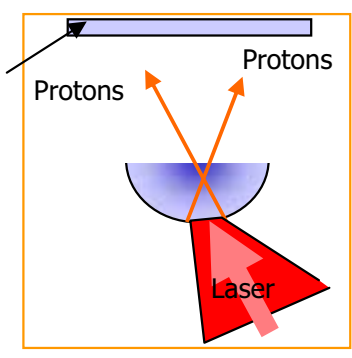

(b) Round

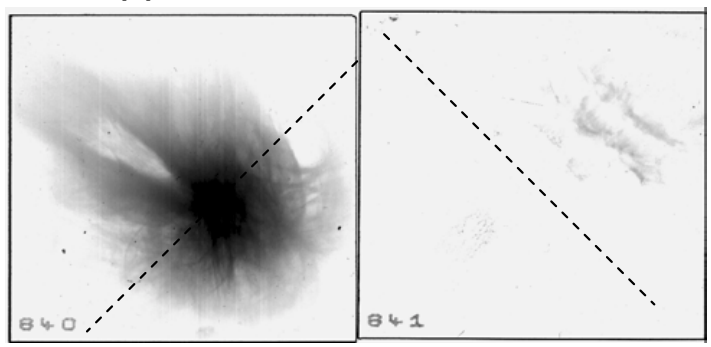

FIGURE 2. 100-120 micron diameter aluminum half-wire target results. Flat side (a) and round side (b) irradiated, protons are recorded on CR-39 numbered 840 (a) and 841 (b). The wires were oriented approximately 45 degrees to the vertical, indicated by dashed lines.

\section{Beam Manipulation}

Much theoretical work on methods to control proton/ion beam production has been introduced in the literature ${ }^{16}$. However, many of these methods require a precision in target control which is not yet achievable. We have taken the idea of micro shaped targets to a macroscopic application. Using a half-wire of between 100-150 microns we observed an effect of the front surface on the produced beam. The laser focal spot was around 10 microns FWHM, with $40 \%$ of the total energy in that area. Fig. 2 shows the results from the aluminum half-wire.

The detector used to record the protons is CR-39, a nuclear track detector $2 \times 2$ inches in area, which is insensitive to x-rays or electrons. Fig. 2 shows the flat surface (a) produces a round beam on CR-39 similar to that of an ordinary flat thin-film target. The round surface (b), however, produces an uncollimated spray of protons on either side of the wire, as would be expected from the cylindrical lens-like surface defocusing the beam.

Also, targets were made from 25 micron aluminum films, shaped to have different radii of curvature by different diameter ball bearings ranging from 0.4 to $1 \mathrm{~mm}$ in diameter. Fig. 3 shows the target geometry (on the left) with an inset image from the $0.2 \mathrm{~mm}$ radius target. The results of firing on the rounded side of two thin-film targets of 0.2 and $0.5 \mathrm{~mm}$ radii of curvature are shown on the right. CR-39 numbers 805 and 793 are beams produced from a target with a radius of $0.2 \mathrm{~mm}$, and numbers 808 and 

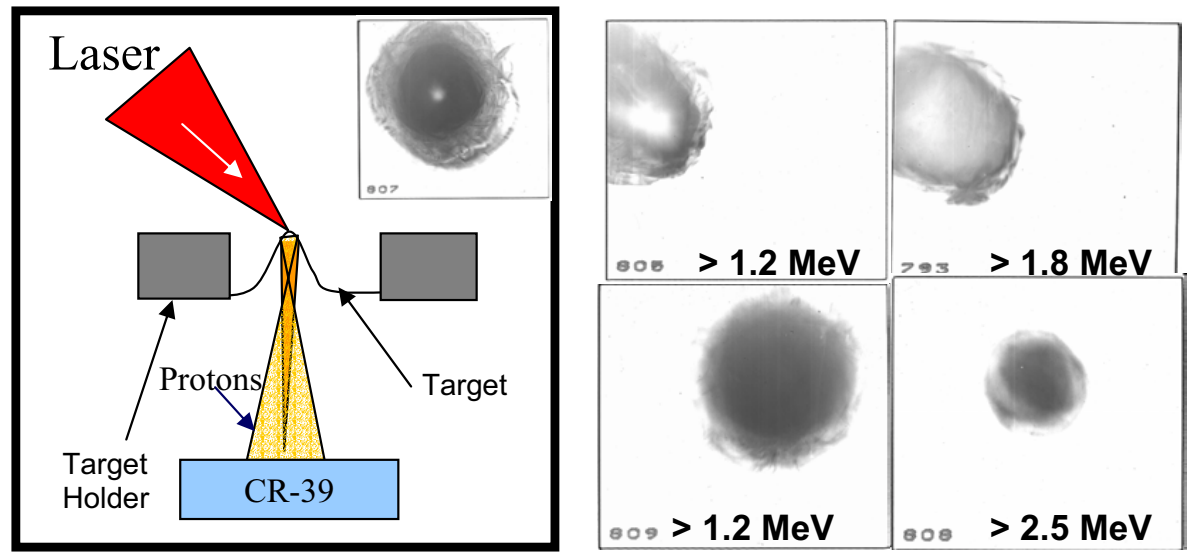

FIGURE 3. Left: Top view of the target geometry is shown, inset of slide 807 shows $>1.8 \mathrm{MeV}$ for $0.2 \mathrm{~mm}$ radius. Right: CR-39 results are shown: 805 and 793 were produced from a $0.2 \mathrm{~mm}$ radius target; 809 and 808 from a $0.5 \mathrm{~mm}$ radius target. All slides are from separate shots.

809 are beams produced from a $0.5 \mathrm{~mm}$ radius target, though each slide is from a separate shot. It must be noted that the CR-39 images here are misleading. In fact what appears to be a ring with a density depression on axis, is actually an oversaturated region in the center and appears brighter due to overlapping tracks. CR39 will become increasingly opaque with increasing track density, until it saturates (at a few $10^{5}$ particles $/ \mathrm{mm}^{2}$ ), then its opacity will decrease. Physical inspection under a microscope reveals the dissimilarity; the myriad of tracks can easily be seen, differentiating it from regions of low track density. The saturated area can then be interpreted as an area of increased flux, which is not seen in a typical beam from a flat target, or in the larger radius targets of slides 808 and 809 . The smaller radius target is harder to align, and this may account for the beam image being off-center on the CR-39. The inset on the left is included to show a strong beam $>1.8$ $\mathrm{MeV}$ from the $0.2 \mathrm{~mm}$ radius at an intensity of nearly $2 \times 10^{19} \mathrm{~W} / \mathrm{cm}^{2}$, here we see clearly the high flux "hole" in the center, and its high degree of symmetry when properly aligned.
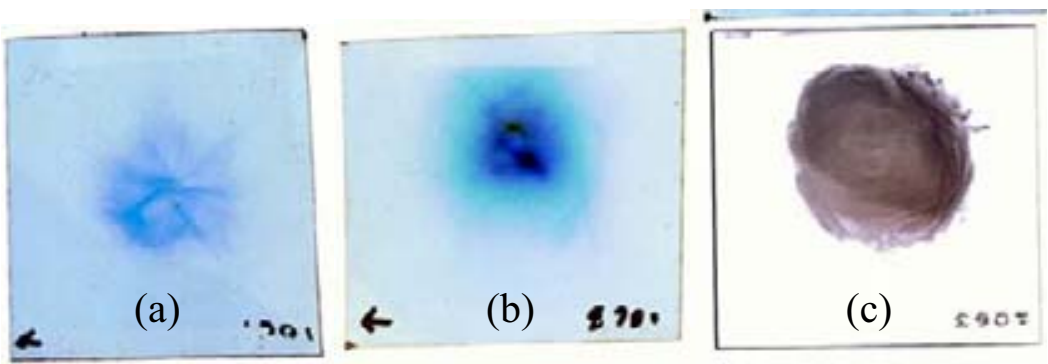

FIGURE 4. Images of beams on radiochromic film from 13 micron Mylar (a) and 12.5 micron aluminum (b) with a subsequent piece of CR-39 (c) from that same shot. 


\section{BEAMS FROM DIELECTRICS AND CONDUCTORS}

A real-time non-destructive diagnostic for the proton beam is being investigated. Currently we are looking at the plasma plume to gain insight into the proton beam's energy and spatial features.

It has been noted that the beam quality varies greatly when using a dielectric versus a conductor. Fig 4 (a) elucidates the difference: a radiochromic film image of a beam from a 13 micron Mylar target, compared to that of (b) a 12.5 micron aluminum target. Radiochromic film has been shown by Clarke et. al. ${ }^{1}$ that the signature of radiochromic film corresponds well with that of the proton beam, as can be seen from our results as well in Fig. 4 (b) and (c) in which a slide of CR-39 (c) was behind the Radiochromic film on the same shot. The Mylar shot (a) shows a remarkable star-like structure, where as the aluminum is fairly uniform. A similarly symmetric pattern is shown on CR-39 from different shot, and different beam energies in Fig 5.

Fig. 5 shows the complicated beam structure which arises from a dielectric target (13 microns of Mylar), and the large difference as compared to the CR-39 in Fig. 4 (c), and also Fig. 3 (808), (809), which, as stated previously, have beam profiles nearly identical to those from flat target beams. The complex structure is thought to arise from huge (30MG) magnetic fields inside the dielectrics ${ }^{17}$.

Not only is the beam quality different, but the maximum proton energies for Mylar and aluminum are about 2 times different. Fig. 6 shows the maximum proton energy versus material thickness for aluminum at the second harmonic (triangles), aluminum at the fundamental (squares), and Mylar at the fundamental (circles). Using the second harmonic of the laser the contrast is 3 orders of magnitude better, but the maximum proton energy is lower. This is due to the need for a certain scale length of pre-plasma before the main pulse arrives. This pre-plasma, created by the foot of the laser pulse, allows for a standing wave in the under dense plasma to heat the electrons, stochastically, to much hotter temperatures ${ }^{18}$, and thus yield hotter protons. The aluminum (triangles) at the second harmonic does not have a peak nor did material choice effect the maximum proton energy, unlike the Mylar (circles) and aluminum (squares) at the fundamental frequency of the laser which both have peaks around 1213 microns and show material dependence on proton energy. The solid line is a calculation taking into account the target thickness and accounting for the proton's

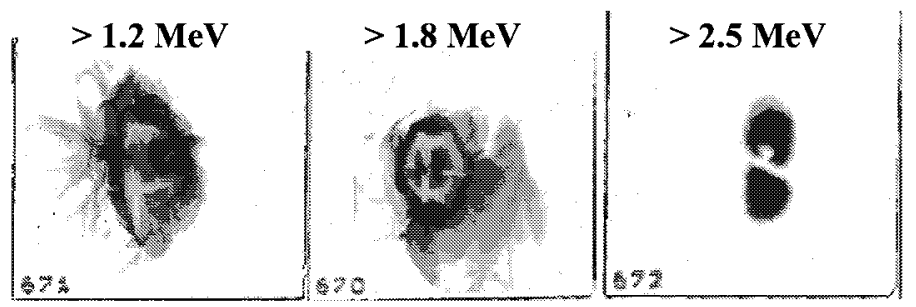

FIGURE 5. Beam images on CR-39 $2 \mathrm{~cm}$ away from a 13 micron Mylar target from 1.2 to $2.5 \mathrm{MeV}$. 


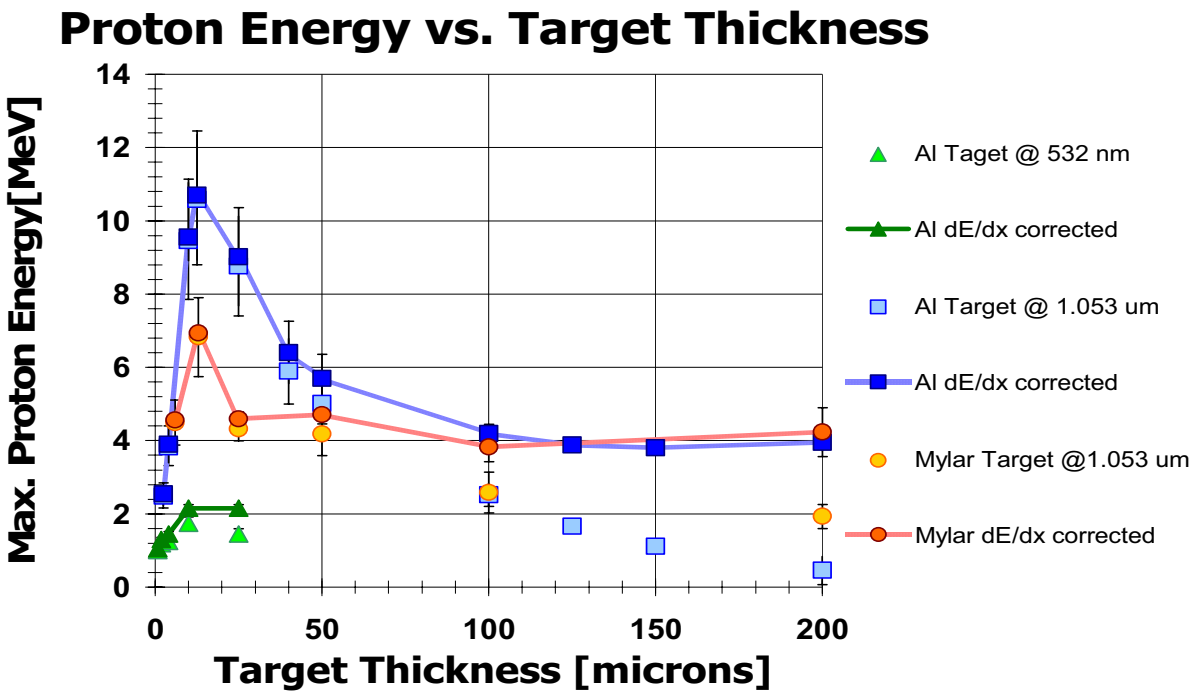

FIGURE 6. Maximum proton energy versus target thickness is shown for aluminum (triangles) at $2 \times 10^{18} \mathrm{~W} / \mathrm{cm} 2$, and Mylar (circles); and aluminum (squares) at $1 \times 10^{19} \mathrm{~W} / \mathrm{cm} 2$. Lines are the calculated energy loss $(\mathrm{dE} / \mathrm{dx})$ correction values for protons having traveled through the target.

energy loss $(\mathrm{dE} / \mathrm{dx})$ through the material with the assumption all originate on the front surface. It is easy to see that the protons from the front surface appear to saturate and plateau at about $4 \mathrm{MeV}$ as the target thickness is increased. We explain the plateau behavior of the energy-loss corrected data as a beam of protons of approximately 4 $\mathrm{MeV}$ produced at the front surface of the target, propagating through the target and losing energy proportional to the distance they travel through the aluminum target.

In contrast to the observed peak Mackinnon $e t . a .^{19}$ report seeing no peak, only a steep decrease in proton energy followed by a much gentler decrease as the thickness increases. The steep decrease is explained by electrons recirculation inside the target during the laser pulse duration $(100 \mathrm{fs})$ which heats them more efficiently. If the target is too thick (about 25 microns) the electrons can not recalculate optimally and do not heat as much, thus the protons are not efficiently accelerated to high energies, and the slope flattens out. An alternative explanation of the nature of the flattened slope is that at the front surface of their targets they are producing a beam of approximately $8.5 \mathrm{MeV}$ protons due to the approximately 10 times greater intensity. This beam then propagates though the target, from 20 to $60 \mathrm{um} \mathrm{8-9} \mathrm{MeV} \mathrm{protons}$ would lose only between 3-6\% of their initial energy, and at 100 um this would increase to about $15 \%$ which would correspond to their data points. It is entirely possible though that some or all of the higher energy protons originate from the rear surface, and the two slopes are an artifact of the two origins of the beams. 


\section{PLUMES FROM DIELECTRICS AND CONDUCTORS}

Laser ablation plumes with longpulse (ns) lasers have been previously well studied theoretically ${ }^{20}$ and experimentally ${ }^{21}$ in the late 70's and 80's. When high power Chirped Pulse Amplified (CPA) picosecond and femtosecond lasers ${ }^{22}$ became widely available in the early 90's solid target interactions and their associated plasma plumes began to be studied extensively both experimentally ${ }^{23}$ and theoretically ${ }^{24}$. In addition, when high-intensity subpicosecond lasers were used, superthermal ions were observed $^{25}$, which are of great interest to the laser deposited materials community. However, the rear-side subpicosecond laser induced back ablation (LIBA) plume, has not been studied in any detail in the high-intensity regime. Some earlier work has been done at lower intensities with nanosecond pulses with thin films supported by transparent substrates ${ }^{26}$, and similar work has been done in the 35 picosecond regime $^{27}$, but the plume itself was not the intended point of study.

We report on the use of the plasma plume integrated optical emission as a diagnostic for thin film properties. The plasma plume geometry and intensity can be used to determine the relative strength of the laser coupling to the material as well as the type of material. The term LIBA can be a confusing term since the proton beam is

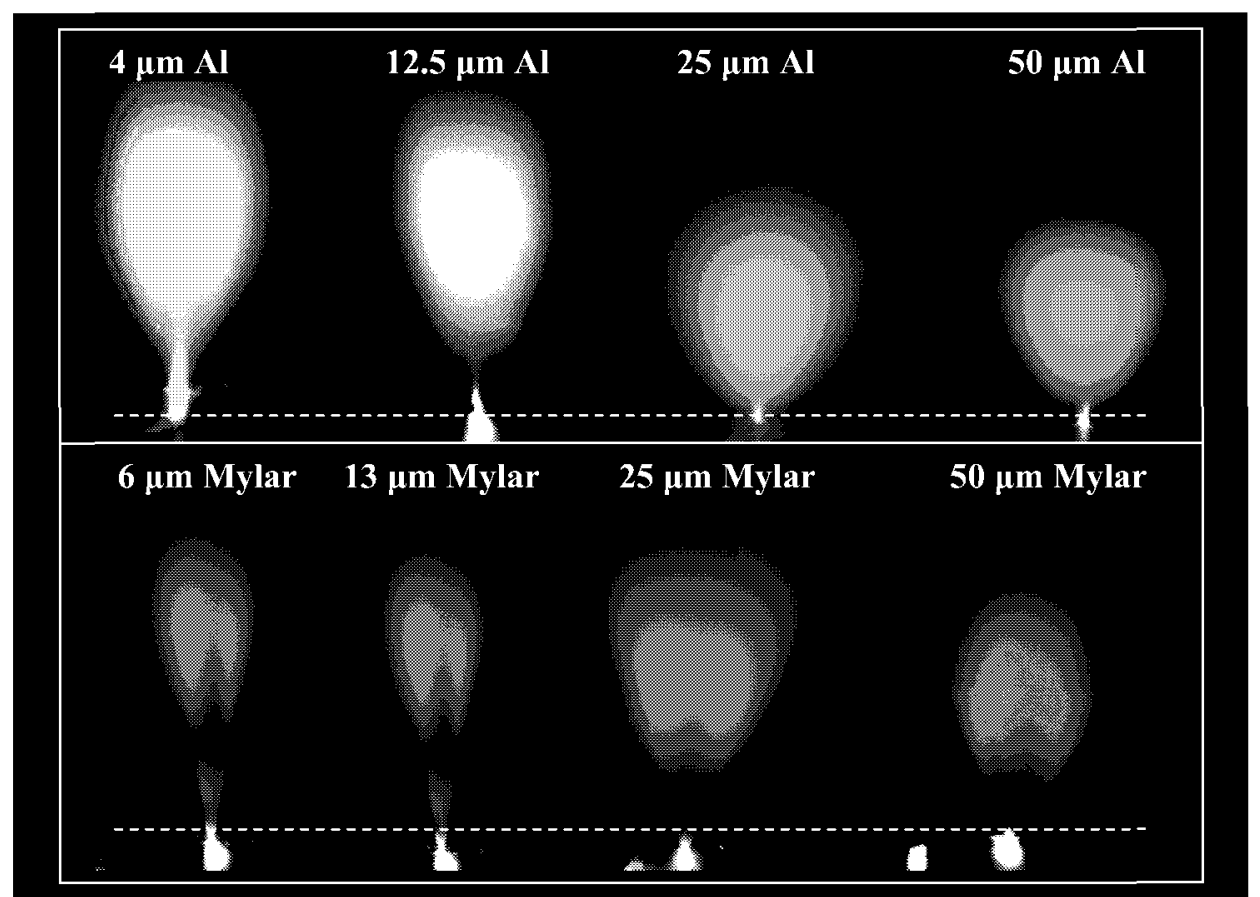

FIGURE 7. Integrated optical plume images for aluminum (top) and Mylar (bottom) for various thicknesses. Plane of the target is out of the page, dashed line indicates target plane, laser is incident from the bottom. Imaged light is from $700 \mathrm{~nm}-350 \mathrm{~nm}$ with a gap of $20 \mathrm{~nm}$ around 532 to block the second harmonic. 
accelerated in the forward direction, hence we shall refer to it as the forward directed ablation plume (FDAP). The FDAP from dielectric targets have marked characteristic differences from that of conductors. Fig. 7 shows the FDAP from aluminum (top) and Mylar (bottom) targets.

From Fig. 7 the difference between the plumes is easy to see, the bright spot is the laser interaction region, and the white dashed line indicates the target plane. The laser is incident from the bottom of the dashed line. The aluminum (top) shows a uniform plume with a tree-like trunk and rounded top structure. The Mylar plumes (bottom) have a unique double lobe feature in the expanding plume, with a dark region between the two lobes, and a fan-like shape. This structure may be due to chemiluminesence in the outer region of the beam as carbon oxidizes, however similar structures have been seen in mostly hydrogen atmospheres.

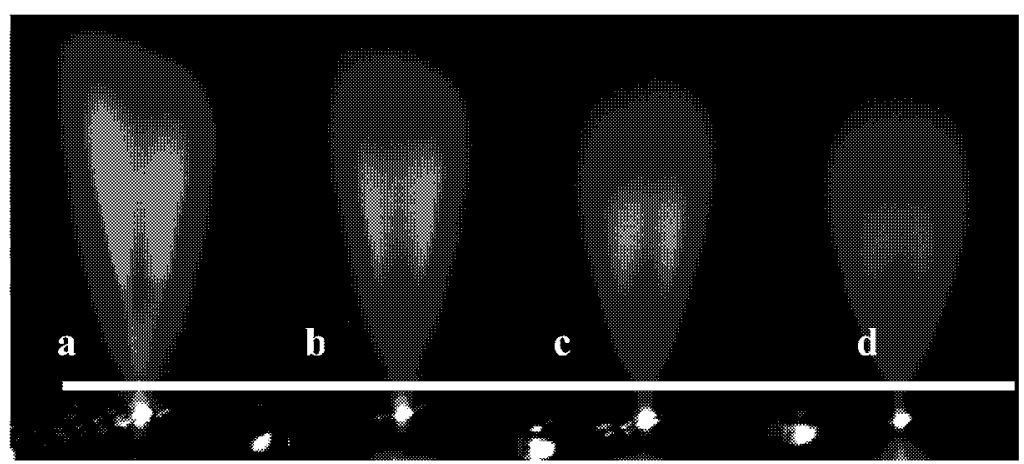

Intensity vs. PIN Diode Output

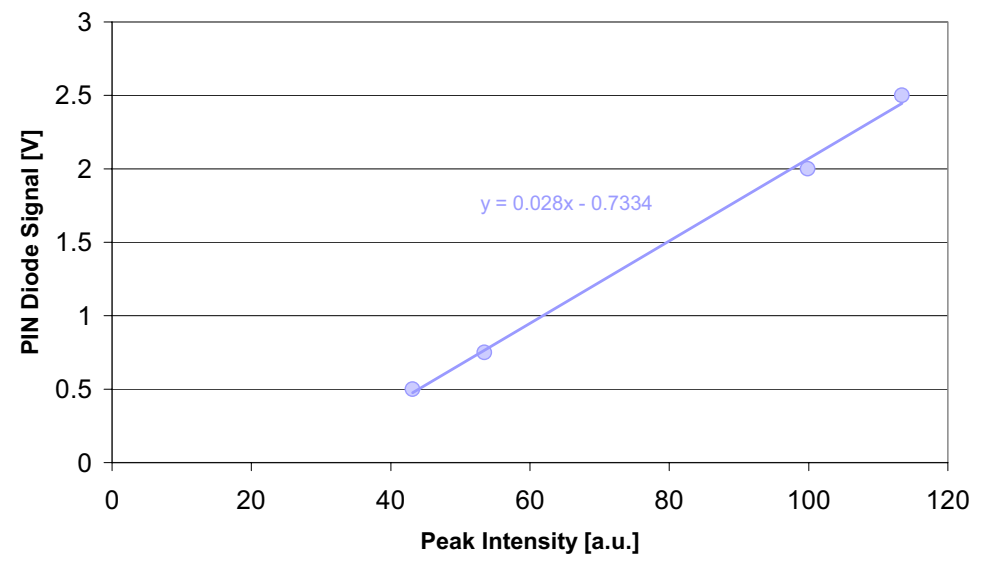

FIGURE 8. At top are 4 images of 13 micron Mylar plumes (a-d) at various PIN diode signal voltages, $2.5 \mathrm{~V}(\mathrm{a}), 2 \mathrm{~V}(\mathrm{~b}), 0.75 \mathrm{~V}$ (c), and $0.5 \mathrm{~V}(\mathrm{~d})$; the graph below shows the correlation between the PIN diode voltage and a 40 -line-averaged line out along the white line. 
A PIN diode is in the chamber to monitor the X-ray signal produced from the hot electrons. We, as well as Badziak ${ }^{28}$, have noted a correlation of the produced $\mathrm{x}$-rays and the proton energy. X-rays are known to correlate with the hot-electron temperature $^{29}$, and the hot-electrons, as discussed above, are responsible for ion acceleration. Thus $\mathrm{x}$-ray PIN diode signals are a main diagnostic of the interaction.

Now, however, we may have a new alternative. Figure 8 shows a set of plumes from 13 micron Mylar for various PIN diode voltages with a 40-line averaged line-out from the area of the plume below the lobed structure as indicated by the white line in Fig. 8. The intensity of this area correlates linearly with the PIN diode signal, which as stated above, correlates with the maximum proton energy. It is our hope that we can refine this technique and implement it as a real time beam diagnostic.

\section{SUMMARY}

We have shown that there exists a beam of protons from high-intensity laser interactions with thin-film targets which originates on the laser irradiated (front) side. We are able to control to some degree the beam by shaping the target's front surface. We have observed a difference in maximum proton energies for Mylar and aluminum, and a peak in the target thickness dependence. We have seen that the difference in proton beam quality from dielectrics and conductors also extends to the forward directed ablation plume, which may be used as a diagnostic for beam energy and quality. Future work entails using the plume to diagnose spatial characteristics of the proton beam.

\section{ACKNOWLEDGMENTS}

This work was supported by Division of High Energy Physics, Office of Energy

Research, U. S. Department of Energy, award DE-FG02-98ER41071, and the National Science Foundation FOCUS Center.

\section{REFERENCES}

1 E. L. Clark et al., Phys. Rev. Lett. 84, 670 (2000); K. Krushelnick et. al., Phys. Plasma 7, 2055 (2000) 2 S. P. Hatchett et al., Phys. Plasma 7, 2076 (2000); R. A. Snavely, Phys. Rev. Lett. 85, 2945 (2000)

3 A. Maksimchuk et. al., Phys. Rev. Lett. 84, 4108 (2000)

4 E.L. Clark, Phys. Rev. Lett. 84, 670 (2000); Y. Murakami et. al., Phys. Plasma 8, 4138 (2001)

5 M. Borgehsi et. al., Phys. Plasma 9, 2214 (2002); J.A. Cobble et. al., J. Appl. Phys. 92, 1775 (2002)

6 S. V. Bulanov and V. S. Khoroshkov, Plasma Phys. Rep. 28, 453 (2002)

7 I. Spencer, "High Intensity Laser Generation of Proton Beams for the Production of $\beta+$ Sources used in Positron Emission Tomography" in Resonance Ionization Spectroscopy 2000: 10th Int'l Symp., edited by J.E. Parks et al., AIP Conference Proceedings 584, New York: American Institute of Physics, 2000, pp. 73-78; M.I.K. Santala, Appl. Phys. Lett. 78, 19 (2001)

8 K. Nemoto et. al., Appl. Phys. Lett. 78, 596 (2001)

9 S.V. Bulanov and V. S. Khoroshkov, Plasma Phys. Rep. 28, 493-496 (2002) 
10 P. A. Norreys, A. P. Fews, F. N. Beg et al., Plasma Phys. Controlled Fusion 40, 175 (1998)

11 V. Yu. Bychenkov et. al., Sov. Phys. JETP 88, 1137 (1999)

12 T. Kammash, K. Flippo, D. Umstadter, "Laser Accelerated Proton Propulsion System (LAPPS)" in AIAA/ASME/SAE/ASEE Joint Propulsion Conference and Exhibit, 36th, 2000

13 W. L. Kruer and K. Estabrook, Phys. Fluids 28, 430 (1985)

14 F. Brunel, Phys. Rev. Lett. 59, 52 (1987)

15 S. C. Wilks et. al. Phys. Plasma 8, 542 (2001)

16 G. S. Sarkisov et. al,. JETP Lett. 69, 20 (1999); S.V. Bulanov et. al., JETP Lett. 71, 401 (2000); H. Ruhlet. Al., Plasma Phys. Rep. 27, 363-371 (2001); A. J. Mackinnon et. al., Phys. Rev. Lett. 88, $215006(2002)$

17 J. R. Davies et al., Phys. Rev. E 56, 7193 (1997)

18 Y. Sentoku et.al., Appl. Phys. B 74, 207 (2002)

19 A. J. Mackinnon et. al., Phys. Rev. Lett. 88, 215006 (2002)

20 L. M.Wickens, J. E. Allen, and P. T. Rumsby, Phys. Rev. Lett. 41, 243 (1978); J. S. Pearlman and R. L. Morse, Phys. Rev. Lett. 40, 1652 (1978)

21 P. M. Campbell et.al., Phys. Rev. Lett. 39, 274 (1977); L. M. Wicknes, J. E. Allen, and P. T. Rumsby, Phys. Rev. Lett. 41, 243 (1978)

22 D. Strickland and G. Mourou, Opt. Commun. 56, 219 (1985)

23 B. Le Drogoff et. al., J. Appl. Phys. 89, 8247 (2001)

24 E. G. Gamaly, Phys. Fluids B 5, 944 (1993)

25 A. P. Fews et. al., Phys. Rev. Lett. 73, 1801 (1994); F. N. Beg e.t al., Phys. Plasma 4, 447 (1997)

26 A. B. Bullock, P. R. Bolton, and F. J. Mayer, J. Appl. Phys. 82, 1828 (1997); P. R. Bolton et. al., J. Opt. Soc. Am. B 13, 336 (1996); J. Bohandy, B. F. Kim, and F. J. Adrian, J. Appl. Phys. 60, 1538 (1986); R. Koppmann, S. M. Refaei, and A. Pospieszcyk, J. Vac. Sci. Technol. A 4, 79 (1986)

27 A. B. Bullock and P.R. Bolton J. Appl. Phys. 85, 460 (1999)

28 J Badziak et. al., Phys. Rev. Lett. 87, 215001 (2001)

29 M. Schnüer, Phys. Plasma 2, 3106 (1995) 